for depression in the medically ill. He claims that satisfactory screening for the symptoms of major depression demonstrates the usefulness of pencil and paper tests to non-psychiatrists. However, the validity of the concept of major depression and the indications for tricyclic drugs in the medically ill with depression have not been satisfactorily established.

Psychological symptoms are distributed continuously in the medical population with no clear separation between the psychiatrically ill and normal subjects. Proportionally more physically ill subjects have high numbers of psychological symptoms because the distribution curve is shifted to the right compared with the community population (House, 1988). A high number of medically ill patients will therefore be diagnosed as suffering from major depression. Since the latter concept was derived from observations of psychiatric patients, it cannot be assumed that a diagnosis of major depression implies the same need for antidepressant treatment in the medically ill. Most cases of significant depression occur because of the patient's awareness of the illness and its implications. Many of these episodes tend to be short-lived, resolve spontaneously (Lloyd \& Cawley, 1983) or require psychosocial forms of treatment. Antidepressants are indicated in the medically ill when major depression precedes a significant medical illness, depressive symptoms are severe, or when they do not resolve after a few months. The role of antidepressants in the treatment of depression in the medically ill is otherwise unclear (Lloyd, 1991) and requires further research.

The specificity and sensitivity of pencil and paper tests for psychiatric disorders for medically ill patients should first be evaluated in terms of prognosis. Tests which identify patients with depression that does not resolve spontaneously after a few months would be extremely useful. Until then, paper and pencil tests for psychiatric disorders have a limited role in clinical practice for non-psychiatrists. There is now good evidence that the detection and management of psychiatric disorders by nonpsychiatrists can be improved by a short course of interviewing training (Gask et al, 1987). This may be a better alternative for improving psychiatric detection and management in the general hospital.

Gask, L., McGrath, G., Goldberg, D. P., et al (1987) Improving the psychiatric skills of established general practitioners: evaluation of group training. Medical Education, 21, 362-368.

HousE, A. (1988) Mood disorders in the physically ill: problems of definition and measurement. Journal of Psychosomatic Research. 32, 345-353.

LloYd, G. G. (1991) Texibook of General Hospital Psychiatry. Edinburgh: Churchill Livingstone.
LLOYD, G. G. \& CAWLEY, R. H. (1983) Distress or illness? A study of psychological symptoms after myocardial infarction. British Journal of Psychiatry, 142, 120-125.

Department of Psychiatry

RICHARD K. MORRISS

University Hospital of South Manchester

West Didsbury

Manchester M20 8LR

\section{Systemic family therapy in adult psychiatry}

SIR: I read with interest the paper by Bloch et al (Journal, September 1991, 159, 357-364). We would agree that there is a need for increased systemic understanding in adult psychiatric practice.

We have had a Brief Therapy clinic in this hospital for three years. Unlike the Milan style favoured by Professor Bloch, we use work derived from the Mental Research Institute and the writings of Steve de Shazer (Fisch et al, 1982; de Shazer, 1988). We have found their approach to be of value in numerous cases. The clinic provides training in systemic work for hospital staff of various disciplines. An outcome study is in progress and early results are promising.

Fisch, R., Weakland, J. H. \& Segal, L. (1982) The Tactics of Change: Doing Therapy Briefly. San Francisco: Jossey-Bass. DE SHAZER, S. (1988) Clues: Investigating Solutions in Brief Therapy. New York: Norton.

Alasdair J. MaCDONAld

Crichton Royal Hospital

Dumfries DGI 4TG

Scotland

\section{Service use by Indian immigrants}

SIR: I read with great interest Sunjai Gupta's paper (Journal, August 1991, 159, 222-225), which is thought-provoking. He does admit the need for further study about the genuine differences in the outcome determinants of psychosis in first-generation Asian immigrants.

I believe that any prospective study in future in relation to service utilisation and determinants of outcome in psychiatric disorders among Asians should take into consideration the "pathway to psychiatric care for Asian patients'. My clinical impression, having worked with a large population of Asians in Leicester, is that almost $30-50 \%$ of my patients, both before and after contact with the psychiatric services, visit traditional healers, hakims, etc. These alternative pathways are pursued either in the UK or abroad. Psychiatric practice in India suggests onethird of patients had treatment with faith healers before the first consultation (Trivedi \& Settu, 1980). 
The need to research this field is paramount in view of increasing reports of 'perceived' or 'real' unmet needs of the mental health of Asians in the UK.

We at Leicester would be keen to set up a joint (bicentre) pilot study with the author to investigate the 'pathways to psychiatric care for Asian patients'. Of the population of the city of Leicester, $25 \%$ is Asian; moreover, a comparison of the first and second generation Asian immigrants would be a useful study to understand the process of acculturation, for which Leicester is such a fertile experimental ground.

TRIVEDI \& SETTU (1980) Healing practices in psychosomatic patients. Indian Journal of Psychiatry, 22, 111.

Department of Psychiatry

DARRYL J. BRITTO

Leicester General Hospital

Gwendolen Road

Leicester LE5 4PW

\section{Eating disorders among Asian girls in Britain}

SIR: Arya (Journal, January 1992, 160, 131-132) attempts to refute the hypothesis that intergenerational conflict contributes to the development of eating disorders among British-born Asian girls in Britain. It is surprising that he does not discuss the evidence presented in our epidemiological study in Bradford (Mumford et al, 1991). At the outset, we had assumed (like Arya) that the most 'Westernised' Asian girls would be at highest risk of developing an eating disorder: the adoption of western cultural values might make them more vulnerable.

However, in our study we found that, on the contrary, it was the Asian girls from most traditional families (as determined by Asian language and dress) who had the highest scores on the Eating Attitudes Test and Body Shape Questionnaire, and who were subsequently diagnosed at interview as having a DSM-III-R eating disorder. The degree of Westernisation (as measured by the use of the English language at home and eating western food) was not related to questionnaire scores nor to the likelihood of receiving an eating-disorder diagnosis.

These findings require an explanation. We have argued that all Asian girls growing up in Britain and attending school here are heavily influenced by prevailing majority cultural values. The girls who experience the most conflict are those who come from the most traditional families, not those from families which have already adopted Western values and outlook.

The specific cultural indicators of intergenerational conflict are likely to vary with different populations. We have repeated our Bradford study in English- medium schools in Lahore, Pakistan (Mumford et al, 1992). Here it was girls who were most 'Westernised' (as measured by the use of the English language at home and a Western diet) who had the highest scores on the Eating Attitudes Test and Body Shape Questionnaire, and who were subsequently diagnosed at interview as having a DSM-III-R eating disorder. Unsurprisingly, responses to items measuring traditional orientation (Asian language and dress) were not related to questionnaire scores nor to the likelihood of receiving an eating-disorder diagnosis, since these are prevailing cultural norms in Lahore.

The hypothesis of intergenerational conflict finds support in studies of adolescents in Kuwait, a conservative Muslim society which was experiencing rapid social change by exposure to Western values, even before the Gulf War. El-Islam et al (1986) found that adolescents' scores on the General Health Questionnaire were associated with greater difference in cultural attitudes between parents and children; there was no relationship between symptoms and 'liberal' attitudes as such.

Intergenerational conflict is widely accepted as a causative factor in many anorexics in Western culture. I regard the tensions which arise from cultural discrepancies between Asian parents and children as merely one specific instance of such conflict. It is highly likely that intergenerational conflict plays a major role in the development of eating disorders among Asian girls in Britain.

El-Islam, M. F., Abu-Dagga, S. I., Malasi, T. H., et al (1986) Intergenerational conflict and psychiatric symptoms. British Journal of Psychiatry, 149, 300-306.

Mumford, D. B., Whitehouse, A. M. \& Platts, M. (1991) Sociocultural correlates of eating disorders among Asian schoolgirls in Bradford. British Journal of Psychiatry, 158, 222-228.

- - \& Choudry, I. V. (1992) A survey of eating disorders in English-medium schools in Lahore (Pakistan). International Journal of Eating Disorders (in press).

Department of Psychiatry

DAVID B. MUMFord

University of Leeds

15 Hyde Terrace

Leeds LS2 9LT

\section{Seasonal risk factors}

SIR: Pulver et al, in their re-examination of the evidence for a seasonal risk factor (Journal, January $1992,160,65-71,71-75)$ in the development of schizophrenia, have neglected to mention several other possible explanations for the trend towards winter/early spring birth dates. Many other conditions whose aetiology is at present unknown have also been recognised as having a similar seasonal 